\title{
Community-acquired pneumonia subgroups and differential response to corticosteroids: a secondary analysis of controlled studies
}

\author{
Esther Wittermans $s^{1,2,12}$, Philip A. van der Zee $\mathbb{1}^{3,12}$, Hongchao $\mathbf{Q i}^{4}$, Ewoudt M.W. van de Garde $\mathbb{C}^{5,6}$, \\ Claudine A. Blum ${ }^{7}$, Mirjam Christ-Crain ${ }^{8}$, Diederik Gommers ${ }^{3}$, Jan C. Grutters ${ }^{9,10}$, G. Paul Voorn ${ }^{11}$, \\ Willem Jan W. Bos ${ }^{1,2}$ and Henrik Endeman ${ }^{3}$
}

\begin{abstract}
${ }^{1}$ Dept of Internal Medicine, St Antonius Hospital, Nieuwegein, The Netherlands. ${ }^{2}$ Dept of Internal Medicine, Leiden University Medical Centre, Leiden, The Netherlands. ${ }^{3}$ Dept of Adult Intensive Care, Erasmus Medical Centre, Rotterdam, The Netherlands. ${ }^{4}$ Dept of Biostatistics, Erasmus Medical Centre, Rotterdam, The Netherlands. ${ }^{5}$ Dept of Clinical Pharmacology, St Antonius Hospital, Nieuwegein, The Netherlands. ${ }^{6}$ Division of Pharmacoepidemiology and Clinical Pharmacology, Faculty of Science, Utrecht University, Utrecht, the Netherlands. ${ }^{7}$ Depts of General Internal and Emergency Medicine, and Endocrinology, Diabetology and Clinical Nutrition, Medical University Clinic, Aarau, Switzerland. ${ }^{8}$ Depts of Endocrinology, Diabetology and Metabolism, and Clinical Research, University Hospital Basel, Basel, Switzerland. ${ }^{9}$ Dept of Pulmonology, St Antonius Hospital, Nieuwegein, The Netherlands. ${ }^{10}$ Division of Heart and Lungs, University Medical Centre Utrecht, Utrecht, The Netherlands. ${ }^{11}$ Dept of Medical Microbiology and Immunology, St Antonius Hospital, Nieuwegein, The Netherlands. ${ }^{12}$ These authors contributed equally.
\end{abstract}

Corresponding author: Philip van der Zee (p.vanderzee@erasmusmc.nl)

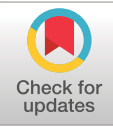

This version is distributed under the terms of the Creative Commons Attribution NonCommercial Licence 4.0. For commercial reproduction rights and permissions contact permissions@ersnet.org

This article has supplementary material available from openres.erjournals.com

Received: 4 Aug 2021 Accepted: 19 Oct 2021

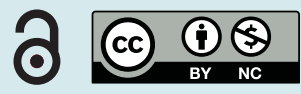

Shareable abstract (@ERSpublications)

Latent class analysis of baseline clinical and biomarker data identified two distinct classes of patients with community-acquired pneumonia in two cohorts. In one cohort, the response to adjunctive corticosteroid treatment differed between classes. https://bit.ly/3CIR92L

Cite this article as: Wittermans E, van der Zee PA, Qi H, et al. Community-acquired pneumonia subgroups and differential response to corticosteroids: a secondary analysis of controlled studies. ERJ Open Res 2022; 8: 00489-2021 [DOI: 10.1183/23120541.00489-2021].

\section{Abstract}

Background Latent class analysis (LCA) has identified subgroups with meaningful treatment implications in acute respiratory distress syndrome. We performed a secondary analysis of three studies to assess whether LCA can identify clinically distinct subgroups in community-acquired pneumonia (CAP) and whether the treatment effect of adjunctive corticosteroids differs between subgroups.

Methods LCA was performed on baseline clinical and biomarker data from the Ovidius trial $(\mathrm{n}=304)$ and the Steroids in Pneumonia (STEP) trial $(n=727)$, both randomised controlled trials investigating adjunctive corticosteroid treatment in CAP, and the observational TripleP cohort $(n=201)$. Analyses were conducted independently in two cohorts (Ovidius-TripleP combined and the STEP trial). In both cohorts, differences in clinical outcomes and response to adjunctive corticosteroid treatment were examined between subgroups identified through LCA.

Results A two-class model fitted both cohorts best. Class 2 patients had more signs of systemic inflammation compared to class 1 . In both cohorts, length of stay was longer and in-hospital mortality rate was higher in class 2. In the Ovidius trial, corticosteroids reduced the median length of stay in class 2 (6.5 versus 9.5 days) but not in class 1 (p-value for interaction=0.02). In the STEP trial, there was no significant interaction for length of stay. We found no significant interaction between class assignment and adjunctive corticosteroid treatment for secondary outcomes.

Conclusions In two independent cohorts, LCA identified two classes of CAP patients with different clinical characteristics and outcomes. Given the different response to adjunctive corticosteroids in the Ovidius trial, LCA might provide a useful basis to improve patient selection for future trials.

Introduction

Treatment of community-acquired pneumonia (CAP) is based on early diagnosis and prompt initiation of antibiotic therapy [1]. Despite effective treatment, CAP remains a leading cause of mortality and morbidity 
worldwide [2]. Adjunctive treatment with corticosteroids might improve clinical outcomes in patients with CAP [3].

A local immune response is crucial to contain and eliminate the primary infection in CAP [4]. However, an uncontrolled or excessive local immune response could result in systemic inflammation and subsequent multi-organ dysfunction [5].

Adjunctive treatment with corticosteroids, a potent inhibitor of the immune response, has shown to reduce length of stay (LOS) and time to clinical stability in hospitalised patients with CAP [3]. However, corticosteroids did not lower the mortality rate, and increased the incidence of hospital readmission and hyperglycaemia requiring insulin therapy [3]. Therefore, treatment guidelines do not recommend routine use of corticosteroids in patients with CAP [1].

In a clinically heterogeneous condition as CAP, it is likely that a subgroup of patients does benefit from corticosteroid treatment [6]. It has been hypothesised that corticosteroid treatment should be given to the subgroup with an excessive systemic inflammation response, whereas patients with a local and controlled immune response should not receive corticosteroid treatment [7]. So far, patients with CAP have been stratified by pneumonia severity index (PSI), initial C-reactive protein concentration, and inflammatory status, but stratification did not result in an unequivocal definition of a subgroup benefiting from corticosteroid therapy and therefore did not result in adjustment of clinical guidelines [3, 8-10].

In other heterogeneous conditions, such as sepsis or acute respiratory distress syndrome, substantial efforts have been made to identify subgroups characterised by different prognoses and responses to treatment [11]. In patients with acute respiratory distress syndrome, a latent class analysis (LCA) was used to identify subgroups with different treatment responses to ventilator and fluid management [12, 13]. The identification of patients that are likely to respond to (corticosteroid) treatment, i.e. predictive enrichment, is a step towards personalised medicine and improved patient selection for future clinical trials [14].

In this secondary analysis of three controlled studies, we attempted to identify CAP subgroups through LCA of baseline clinical and biomarker data from two randomised controlled trials and one prospective cohort study. In addition, we examined whether LCA-based subgroups were associated with different clinical outcomes and a different response to adjunctive corticosteroids.

Materials and methods

Study population and study design

This is a secondary analysis of demographic, clinical and biomarker data obtained at baseline from patients enrolled in the observational TripleP cohort [15], and two multicentre randomised controlled trials: the Ovidius trial (NCT00471640) [16] and the Steroids in Pneumonia (STEP) trial (NCT00973154) [17]. All studies included hospitalised adult patients with CAP (see supplementary materials).

In the Ovidius trial, patients with CAP were randomly allocated to receive intravenous dexamethasone $5 \mathrm{mg}$ daily or placebo for 4 days following hospital admission [16]. The STEP trial randomised 727 patients with CAP to either placebo or oral prednisolone $50 \mathrm{mg}$ daily for 7 days in the per protocol analysis [17]. LOS, the primary endpoint in the Ovidius trial and main secondary endpoint in the STEP trial, was significantly reduced in patients assigned to adjunctive treatment with corticosteroids. Details of the original studies are published elsewhere $[16,17]$.

The Ovidius trial and TripleP study were approved by the Medical Ethics Committee at the St Antonius Hospital. The ethical committees of all participating hospitals and Swissmedic approved the STEP trial.

\section{Methods}

Two separate LCAs were performed for the identification of subgroups: one in a combined cohort of TripleP and the Ovidius trial, and one in the STEP trial. The observational TripleP cohort $(n=201)$ and the Ovidius trial $(n=304)$ were combined to obtain a larger sample size. We chose to combine these cohorts as the TripleP cohort preceded the Ovidius trial and reported similar clinical and biomarker data. The Ovidius trial and TripleP study are two mutually exclusive cohorts. The STEP trial $(n=727)$ was analysed independently as different clinical and biomarker data were recorded.

After identification of subgroups by LCA, differences in clinical outcomes between these subgroups and the presence of interaction between treatment allocation and LCA-defined subgroups were assessed separately in both cohorts (Ovidius-TripleP combined and STEP). For the Ovidius-TripleP cohort, only 
patients who participated in the Ovidius trial were included in the analysis of the interaction between adjunctive corticosteroids. The primary outcome was LOS and secondary outcomes were intensive care unit (ICU) admission, in-hospital mortality, 30-day mortality and 30-day hospital readmission.

\section{Statistical analysis}

Baseline characteristics of the Ovidius-TripleP combined and STEP cohorts were presented as count (\%) for categorical variables, and mean (standard deviation) or median (interquartile range, IQR) for continuous variables, after testing for normal distribution. Baseline characteristics of both cohorts were compared using an independent samples t-test, Mann-Whitney U test or Chi-squared test, as appropriate.

The DepmixS4 package in R 4.0.0 (R core team, 2020) was used to conduct the LCA. Baseline clinical and biomarker data obtained at hospital admission were used as class-defining variables in the LCA. A full list of class-defining variables included in the LCA for each cohort is shown in the supplementary material. Assignment of patients to classes was performed independently of clinical outcomes. LCA was first conducted in the Ovidius-TripleP cohort, and was repeated independently in the STEP cohort. Missing data were accommodated by estimating model parameters based on the full information maximum likelihood [18].

We fitted models with latent classes ranging from two to five classes. To determine the best-fitting model, we used the following criteria: 1) clinical interpretability, i.e. whether identified classes corresponded to clinically coherent clusters of clinical and biomarker data; 2) the number of patients assigned to the smallest class, where a model with small class size is statistically less meaningful; and 3) the Bayesian information criterion, where a lower number corresponds with improved model fit. For clinical interpretability, all continuous variables in the LCA were rescaled to a z-scale with a mean of zero and standard deviation of 1 . Subsequently, clinical interpretability was assessed by two authors independently ( $\mathrm{PZ}$ and HE). Discrepancies were resolved by consensus and, if necessary, a third author was consulted.

Once the number of classes was determined, patients were assigned to the class with maximum probability of class assignment based on the LCA model. The probability of a patient being assigned to a specific class is a weighted average of the $\mathrm{N}$ class-specific probabilities in LCA, so each patient has probabilities assigned to all classes, respectively. For example, a patient with a probability of $90 \%$ to be assigned to class 1 and $10 \%$ probability to be assigned to class 2 was assigned to class 1 . Subsequently, the association between class assignment and baseline characteristics or clinical outcomes was tested using Chi-squared, Mann-Whitney U or independent samples t-test, as appropriate. Finally, for the Ovidius trial and STEP cohorts, we tested the interaction between randomly assigned treatment and class on clinical outcomes with the Poisson regression model for LOS and Chi-squared test for categorical outcomes. A p-value $<0.05$ was deemed statistically significant.

\section{Results}

Baseline characteristics

Baseline characteristics of both cohorts are presented in table 1 and supplemental table e1. In short, patients in the Ovidius-TripleP cohort were younger, had fewer comorbidities and had higher levels of inflammatory biomarkers as compared to patients in the STEP cohort. LOS was longer in the OvidiusTripleP cohort as compared to the STEP cohort (8.5; 6.0-13.0 days versus 7.0; 4.0-10.0 days, p-value $<0.001)$. Secondary outcomes were similar between both cohorts.

\section{Latent class modelling: identification of number of classes}

We fitted latent class models ranging from two to five classes (table 2). First, we examined clinical interpretability by plotting class-defining variables for all models and assessed whether identified classes corresponded to clinically coherent subgroups (figure 1 and supplemental figure e1). In both the OvidiusTripleP cohort and the STEP cohort, a two-class model resulted in two coherent and distinct clinical classes. Addition of a third, fourth or fifth class resulted in further subdivision of patients assigned to class 2 in the two-class model, without adding an additional coherent or distinct clinical class. Subsequently, we explored the number of patients per subgroup in all models (table 2). The addition of a third class to the two-class model resulted in a smaller third class of 58 patients in the Ovidius-TripleP cohort and 72 patients in the STEP cohort. We observed a further decline in the number of patients in the smallest class in a four- or five-class model. Lastly, the Bayesian information criterion was lowest in the five-class model in both the Ovidius-TripleP cohort and the STEP cohort, suggesting a better fit for the five-class model. Even though a data-driven approach suggested more than two classes, a three-class model did not result in an evident third clinical entity. Thus, clinical interpretability of the two-class models in conjunction with the relatively small number of patients in the three-, four- or five-class models led us to proceed with the 


\begin{tabular}{|c|c|c|}
\hline & Ovidius-TripleP cohort $(n=505)$ & STEP cohort $(n=727)$ \\
\hline \multicolumn{3}{|l|}{ Demographic data } \\
\hline Age (years) & $67(51-78)$ & $73(60-83)$ \\
\hline Male & $295(58.4 \%)$ & $452(62.2 \%)$ \\
\hline Caucasian & $491(97.2 \%)$ & $712(97.9 \%)$ \\
\hline Duration of symptoms (days) & $4(2-7)$ & $4(2-7)$ \\
\hline Antibiotics at home & $130(25.7 \%)$ & $164(22.6 \%)$ \\
\hline Corticosteroids at home & $34(6.7 \%)$ & $14(1.9 \%)$ \\
\hline \multicolumn{3}{|l|}{ Comorbidities } \\
\hline Nursing home resident & $19(3.8 \%)$ & $0(0.0 \%)$ \\
\hline Cerebrovascular accident & $46(9.1 \%)$ & $67(9.2 \%)$ \\
\hline Malignancy & $45(8.9 \%)$ & $70(9.6 \%)$ \\
\hline Liver disease & $2(0.4 \%)$ & $28(3.9 \%)$ \\
\hline Renal disease & $40(7.9 \%)$ & $218(30.0 \%)$ \\
\hline Congestive heart failure & $68(13.5 \%)$ & $134(18.4 \%)$ \\
\hline Chronic obstructive pulmonary disease & $98(19.4 \%)$ & $122(16.8 \%)$ \\
\hline Diabetes mellitus & $77(15.2 \%)$ & $139(19.1 \%)$ \\
\hline Current smoker & $81(16.0 \%)$ & $188(25.9 \%)$ \\
\hline Pneumonia severity index score & $87(63-114)$ & $90(64-113)$ \\
\hline \multicolumn{3}{|l|}{ Outcome } \\
\hline Length of stay (days) & $8.5(6.0-13.0)$ & $7.0(4.0-10.0)$ \\
\hline ICU admission & $38(7.5 \%)$ & $39(5.4 \%)$ \\
\hline In-hospital mortality & $24(4.8 \%)$ & $24(3.3 \%)$ \\
\hline 30-day mortality & $26(5.1 \%)$ & $28(3.9 \%)$ \\
\hline Readmission & $37(7.3 \%)$ & $39(5.4 \%)$ \\
\hline
\end{tabular}

Data are $\mathrm{n}(\%)$, mean (standard deviation) or median (interquartile range). ICU: intensive care unit; STEP: Steroids in Pneumonia.

two-class models for both cohorts. We will refer to the classes as class 1 and class 2 in the remainder of the manuscript. For the three-class model we show clinical characteristics for each class in the supplementary material.

Patients were assigned to the class for which the probability of belonging to that class was the highest. Thus, all patients in both cohorts were assigned to either class 1 or class 2 . In the Ovidius-TripleP cohort, 411 patients were assigned to class 1 and 94 to class 2. In the STEP cohort, 574 and 153 patients were assigned to class 1 and class 2, respectively. Probabilities of class assignment for the two-class model are presented in supplemental figure e2. The average probability of a patient belonging to the class to which it was assigned was $99.4 \%$ for class 1 and 98.6\% class 2 in the Ovidius-TripleP cohort, and 98.7\% for class 1 and $99.1 \%$ for class 2 in the STEP cohort. This indicated a good model fit and robust class assignment.

\section{TABLE 2 Fit statistics for latent class models from two to five class models}

\begin{tabular}{|c|c|c|c|c|c|c|}
\hline \multirow[t]{2}{*}{ Number of classes } & \multirow[t]{2}{*}{$\mathrm{BIC}$} & \multicolumn{5}{|c|}{ Number of patients per class } \\
\hline & & 1 & 2 & 3 & 4 & 5 \\
\hline \multicolumn{7}{|c|}{ Ovidius-TripleP cohort } \\
\hline 2 & 124577.2 & 411 & 94 & & & \\
\hline 3 & 120741.9 & 153 & 58 & 294 & & \\
\hline 4 & 120507.3 & 61 & 112 & 296 & 36 & \\
\hline 5 & 118372.7 & 33 & 25 & 94 & 108 & 245 \\
\hline \multicolumn{7}{|l|}{ STEP cohort } \\
\hline 2 & 116815.7 & 574 & 153 & & & \\
\hline 3 & 106770.5 & 99 & 556 & 72 & & \\
\hline 4 & 71445.1 & 24 & 125 & 466 & 112 & \\
\hline 5 & 70684.5 & 132 & 18 & 44 & 434 & 99 \\
\hline
\end{tabular}



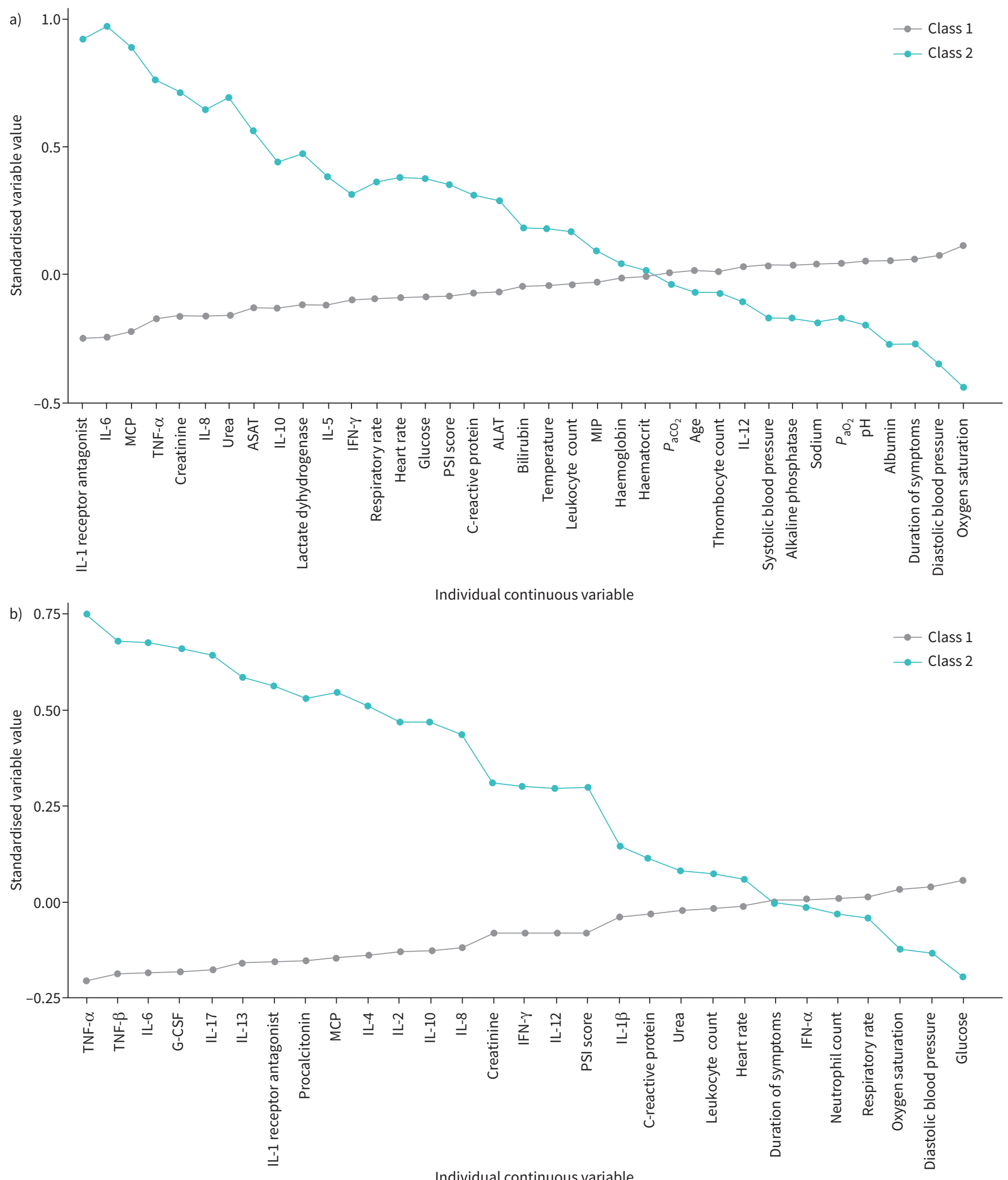

FIGURE. 1 Continuous variables (standardised) by class assignment for the a) Ovidius-TripleP cohort and b) Steroids in Pneumonia (STEP) cohort. Differences between the standardised values of each variable by class ( $y$-axis) for the variable shown on the $x$-axis. The variables are sorted by degree of separation between classes: from the maximum positive separation on the left (where the standardised value of class 2 is higher than the standardised value of class 1 ) to the maximum negative separation on the right (where the standardised value of class 2 to is lower than the standardised value of class 1 ). The crossover of the lines indicates that the standardised value for this variable was the same for classes 1 and 2 
(i.e. no difference between class 1 and class 2 for this variable). Therefore, variables near the intersection of both lines are similar in both classes and thus are not class-defining. The method of variable standardisation is described in the methods section. If the standardised value of a certain variable is 1 for a class, it means that the mean value for that variable within that class was one standard deviation higher than the mean value for that variable in the whole cohort. LAT: alanine transaminase; ASAT: aspartate transaminase; G-CSF: granulocyte colony-stimulating factor; IFN: interferon; IL: interleukin; MCP: monocyte chemoattractant protein; MIP:macrophage inflammatory protein; $P_{\text {aco }}$ : arterial carbon dioxide tension; $P_{\mathrm{aO}_{2}}$ : arterial oxygen tension; PSI: pneumonia severity index; TNF: tumour necrosis factor.

\section{Class characteristics}

Differences between class 1 and class 2 in the Ovidius-TripleP cohort are shown in figure 1a and table 3. The most noteworthy and clinically relevant differences were that patients in class 2 had higher plasma concentration of interleukin (IL)-1 receptor antagonist, IL-6, monocyte chemoattractant protein and tumour necrosis factor- $\alpha$ (TNF- $\alpha$ ) compared to class 1 . Furthermore, patients assigned to class 2 seemed to have more severe illness seeing as they had lower oxygen saturation, lower diastolic blood pressure and had a higher PSI score at admission.

Differences between class 1 and class 2 in the STEP cohort are shown in figure $1 \mathrm{~b}$ and table 4 . In the STEP cohort, the most noteworthy and clinically relevant differences between classes were higher plasma concentrations of TNF- $\alpha$, interferon- $\beta$, IL-6, granulocyte colony stimulating factor and IL-17 in class 2 compared to class 1 . Patients in class 2 also had a higher PSI score compared to class 1 . However, there was no difference in oxygen saturation or diastolic blood pressure.

\section{Class prediction with a small number of variables}

In order to determine whether classes could be identified based on a reduced number of variables, we tested a three-variable model including variables available for both cohorts and differing most between classes (IL-6, TNF- $\alpha$ and oxygen saturation at hospital admission). An area under the curve (AUC) was calculated to evaluate this reduced model compared to the full model. The AUC was 0.78 and 0.65 , respectively, for the Ovidius-TripleP cohort and the STEP cohort. Contingency tables comparing class membership between reduced and full model are shown in the supplementary material (table e2).

\section{Association between class and clinical outcomes}

Subsequently, we assessed clinical outcomes in both classes (table 5). In the Ovidius-TripleP cohort, patients in class 2 had a significantly longer LOS (10.5; 6.5-16.0 days versus 8.0; 6.0-12.0 days, p-value $<0.01$ ) and higher rate of ICU admissions. In-hospital mortality and 30-day mortality rates were significantly higher in class 2. Similar results were observed in the STEP cohort, as patients in class 2 had a longer LOS (7.0; 5.0-12.0 days versus 7.0; 4.0-10.0 days, p-value <0.01), and a higher in-hospital mortality rate (table 5).

\section{Effect of corticosteroids on outcome stratified by class}

Lastly, we used the data from the Ovidius trial and the STEP cohort to determine whether classes responded differently to randomly assigned adjunctive treatment with corticosteroids (table 6). In the Ovidius trial, dexamethasone reduced LOS in patients assigned to class 2 (6.5; 5.5-10.0 days versus 9.5; 5.0-14.5 days), whereas LOS was similar between treatment groups in class 1 ( $\mathrm{p}$-value for interaction 0.02). In the STEP cohort, there was no significant interaction for LOS between class assignment and adjunctive treatment with corticosteroids. In both cohorts, we found no significant interaction for secondary outcomes between class assignment and adjunctive treatment with corticosteroids.

\section{Discussion}

In this secondary analysis of three controlled studies, LCA identified two distinct classes of CAP patients with different biomarker profiles, clinical characteristics and clinical outcomes. Classes were identified in two independent cohorts, despite multiple significant differences in baseline characteristics between cohorts. In the Ovidius trial, adjunctive treatment with corticosteroids reduced LOS only in patients assigned to class 2. We found no differential treatment response for LOS in the STEP cohort or for secondary outcomes in both cohorts.

In both cohorts, class 2 was characterised by higher concentrations of inflammatory biomarkers, creatinine and higher PSI scores. Additionally, patients assigned to class 2 in the Ovidius-TripleP cohort had lower oxygen saturation, lower diastolic blood pressure and higher incidence of oxygen therapy. In contrast, patients in class 1 were characterised by lower concentrations of inflammatory plasma biomarkers and 
TABLE 3 Values of variables at baseline stratified by class in the Ovidius-TripleP cohort

\begin{tabular}{|c|c|c|c|}
\hline Variable & Class $1(n=411)$ & Class $2(n=94)$ & Missing $\mathrm{n}(\%)$ \\
\hline Age (years) & $67(51-79)$ & $67(53-76)$ & $0(0)$ \\
\hline Alanine transaminase $\left(\mathrm{U} \cdot \mathrm{L}^{-1}\right)$ & $28(16-44)$ & $28(19-55)$ & $152(30.1)$ \\
\hline Albumin $\left(g \cdot \mathrm{L}^{-1}\right)$ & $37(33-40)$ & $36(33-38)$ & $339(67.0)$ \\
\hline Alkaline phosphatase $\left(\mathrm{U} \cdot \mathrm{L}^{-1}\right)$ & $90(70-130)$ & $90(61-113)$ & $167(33.1)$ \\
\hline Altered mental status & $47(11.4)$ & $10(10.6)$ & $11(2.2)$ \\
\hline Aspartate transaminase $\left(\mathrm{U} \cdot \mathrm{L}^{-1}\right)$ & $34(23-51)$ & $38(25-78)^{\#}$ & $153(30.3)$ \\
\hline Bilirubin $\left(\mu \mathrm{mol} \cdot \mathrm{L}^{-1}\right)$ & $12(9-16)$ & $16(12-24)^{\#}$ & $199(39.4)$ \\
\hline $\mathrm{C}$-reactive protein $\left(\mathrm{mg} \cdot \mathrm{L}^{-1}\right)$ & $196(94-300)$ & $294(107-389)^{\#}$ & $9(1.8)$ \\
\hline Cortisol $\left(\mathrm{nmol}^{\circ} \mathrm{L}^{-1}\right)^{q}$ & $226.0(148.0-159.1)$ & $446.8(322.4-691.4)^{\#}$ & $23(4.6)$ \\
\hline Corticosteroids at home & $30(7.5)$ & $4(4.4)$ & $15(3.0)$ \\
\hline Creatinine $\left(\mu \mathrm{mol} \cdot \mathrm{L}^{-1}\right)$ & $84(70-106)$ & $111(91-157)^{\#}$ & $10(2.0)$ \\
\hline Diastolic blood pressure (mmHg) & $75(68-83)$ & $70(60-80)^{\#}$ & $11(2.2)$ \\
\hline Duration of symptoms (days) & $4(3-7)$ & $3(2-5)^{\#}$ & $16(3.2)$ \\
\hline Glucose $\left(\mathrm{mmol} \cdot \mathrm{L}^{-1}\right)$ & $7.0(6.0-8.3)$ & $7.5(6.2-9.8)^{\#}$ & $39(7.7)$ \\
\hline Heart rate (beats $\cdot \mathrm{min}^{-1}$ ) & $95(82-109)$ & $110(87-118)^{\#}$ & $9(1.8)$ \\
\hline Haematocrit $\left(L \cdot L^{-1}\right)$ & $0.40(0.36-0.43)$ & $0.39(0.37-0.43)$ & $17(3.4)$ \\
\hline Haemoglobin $\left(\mathrm{mmol}^{\circ} \mathrm{L}^{-1}\right)$ & $8.3(7.6-9.0)$ & $8.3(7.8-9.0)$ & $10(2.0)$ \\
\hline Interferon- $\gamma\left(\mathrm{pg} \cdot \mathrm{mL}^{-1}\right)$ & $202.1(16.8-288.3)$ & $217.8(10.0-354.9)$ & $213(42.2)$ \\
\hline Interleukin-1 receptor antagonist $\left(\mathrm{pg} \cdot \mathrm{mL}^{-1}\right)$ & $102.8(18.0-448.4)$ & $1042.5(204.2-4309.2)^{\#}$ & $79(15.6)$ \\
\hline Interleukin- $6\left(\mathrm{pg} \cdot \mathrm{mL}^{-1}\right)$ & $51.0(18.0-156.3)$ & $749.7(101.2-2209.7)^{\#}$ & $63(12.5)$ \\
\hline Interleukin-5 (pg.mL $\left.{ }^{-1}\right)$ & $0.54(0.24-0.77)$ & $0.46(0.26-0.61)$ & $333(65.9)$ \\
\hline Interleukin-8 (pg:mL $\left.{ }^{-1}\right)$ & $14.8(8.1-29.3)$ & $59.5(32.1-152.2)^{\#}$ & $56(11.1)$ \\
\hline Interleukin-10 (pg-mL $\left.{ }^{-1}\right)$ & $3.4(1.4-9.0)$ & $15.9(5.8-79.7)^{\#}$ & $94(18.6)$ \\
\hline Interleukin-12 $\left(\mathrm{pg} \cdot \mathrm{mL}^{-1}\right)$ & $7.3(4.1-10.5)$ & $8.3(5.6-11.5)$ & $337(66.7)$ \\
\hline Lactate dehydrogenase $\left(\mathrm{U} \cdot \mathrm{L}^{-1}\right)$ & $328(252-480)$ & $435(313-604)^{\#}$ & $212(42.0)$ \\
\hline Legionella species ${ }^{4}$ & $14(3.4)$ & $6(6.4)$ & $0(0)$ \\
\hline Leukocyte count $\left(10^{9}\right.$ cells $\left.\cdot \mathrm{L}^{-1}\right)$ & $13.5(9.5-17.7)$ & $14.9(10.8-20.1)$ & $9(1.8)$ \\
\hline Macrophage inflammatory protein $\left(\mathrm{pg} \cdot \mathrm{mL}^{-1}\right)$ & $6.1(3.7-8.5)$ & $6.8(4.6-10.4)$ & $236(47)$ \\
\hline Male $^{\text {q }}$ & $236(57.4)$ & $59(62.8)$ & $0(0)$ \\
\hline Monocyte chemoattractant protein $\left(\mathrm{pg} \cdot \mathrm{mL}^{-1}\right)$ & $274.2(74.7-536.6)$ & $918.4(242.9-2463.3)^{\#}$ & $46(9.1)$ \\
\hline Oxygen saturation (\%) & $94(92-97)$ & $94(88-96)^{\#}$ & $107(21.2)$ \\
\hline Oxygen therapy & $70(17.0)$ & $30(31.9)^{\#}$ & $312(61.8)$ \\
\hline$P_{\mathrm{aO}_{2}}(\mathrm{kPa})$ & $8.80(7.80-10.38)$ & $8.40(7.10-9.90)^{\#}$ & $124(24.6)$ \\
\hline$P_{\mathrm{aCO}_{2}}(\mathrm{kPa})$ & $4.40(4.10-4.90)$ & $4.40(4.00-4.85)$ & $124(24.6)$ \\
\hline $\mathrm{pH}$ & $7.47(7.44-7.50)$ & $7.46(7.42-7.49)$ & $124(24.6)$ \\
\hline Pleural effusion" & $61(14.8)$ & $21(22.3)$ & $9(1.8)$ \\
\hline Pneumonia severity index score & $84(60-111)$ & $102(73-126)^{\#}$ & $0(0)$ \\
\hline Respiratory rate (breaths $\cdot \mathrm{min}^{-1}$ ) & $22(18-30)$ & $25(20-30)^{\#}$ & $104(20.6)$ \\
\hline Sodium $\left(\mathrm{mmol} \cdot \mathrm{L}^{-1}\right)$ & $135(132-137)$ & $133(129-137)^{\#}$ & $9(1.8)$ \\
\hline Streptococcus pneumoniae & $85(20.7)$ & $39(41.5)^{\#}$ & $0(0)$ \\
\hline Systolic blood pressure (mmHg) & $131(120-146)$ & $126(112-145)$ & $11(2.2)$ \\
\hline Temperature $\left({ }^{\circ} \mathrm{C}\right)$ & $38.2(37.4-39.0)$ & $38.5(37.4-39.3)$ & $9(1.8)$ \\
\hline Thrombocyte count $\left(10^{9}\right.$ cells $\left.\cdot \mathrm{L}^{-1}\right)$ & $253(200-317)$ & $237(177-327)$ & $9(1.8)$ \\
\hline Tumour necrosis factor- $\alpha\left(\mathrm{pg} \cdot \mathrm{mL}^{-1}\right)$ & $5.9(3.1-10.2)$ & $12.4(6.1-29.6)^{\#}$ & $224(44.4)$ \\
\hline Urea $\left(\mathrm{mmol} \cdot \mathrm{L}^{-1}\right)$ & $6.4(4.6-9.5)$ & $9.8(6.3-15.2)^{\#}$ & $17(3.4)$ \\
\hline
\end{tabular}

Data are shown as median (interquartile range) or $\mathrm{n}(\%)$. \#: statistically significant difference between class 1 and class 2 . ॥: non-class-defining variables (variable not included in latent class analysis). Missing data is $n(\%)$ for the whole cohort.

lower PSI scores. Furthermore, in the Ovidius-TripleP cohort, cortisol was also higher in class 2 compared to class 1; we assume this is explained by the fact patients with more inflammation have an higher activation of the hypothalamic-pituitary-adrenal axis and thus higher cortisol levels than patients with lower levels of systemic inflammation because they are more severely ill. Moreover, in both cohorts, LOS was longer, and incidence of ICU admissions and mortality rates were higher in class 2. Thus, patients in class 2 had a stronger systemic inflammatory response, whereas patients in class 1 had fewer signs of systemic inflammation. Patients in class 2 were more likely to benefit from the anti-inflammatory effects of corticosteroids, whereas the patients assigned to class 1 were less likely to benefit from the anti-inflammatory effects, at a similar risk of adverse effects. 
TABLE 4 Values of variables at baseline stratified by class in the Steroids in Pneumonia (STEP) cohort

\begin{tabular}{|c|c|c|c|}
\hline Variable & Class $1(n=574)$ & Class $2(n=153)$ & $\begin{array}{l}\text { No. missing } \mathrm{n} \\
(\%)\end{array}$ \\
\hline Altered mental status ${ }^{\natural}$ & $33(5.7)$ & $13(8.5)$ & $0(0)$ \\
\hline C-reactive protein $\left(\mathrm{mg} \cdot \mathrm{L}^{-1}\right)$ & $155(74-247)$ & $171(93-268)$ & $7(1)$ \\
\hline Creatinine $\left(\mu \mathrm{mol} \cdot \mathrm{L}^{-1}\right)$ & $86(68-109)$ & $98(72-132)^{\#}$ & $6(0.8)$ \\
\hline Diastolic blood pressure (mmHg) & $70(60-78)$ & $66(59-75)$ & $4(0.6)$ \\
\hline Duration of symptoms (days) & $4(2-7)$ & $4(2-7)$ & $17(2.3)$ \\
\hline Glucose $\left(\mathrm{mmol} \cdot \mathrm{L}^{-1}\right)$ & $6.4(5.5-7.7)$ & $6.0(5.5-7.3)$ & $179(24.6)$ \\
\hline $\begin{array}{l}\text { Granulocyte colony stimulating factor } \\
\left(\mathrm{pg} \cdot \mathrm{mL}^{-1}\right)\end{array}$ & $7.0(7.0-8.7)$ & $21.1(9.3-59.3)^{\#}$ & $55(7.6)$ \\
\hline Heart rate (beats $\cdot \mathrm{min}^{-1}$ ) & $83(72-95)$ & $84(71-101)$ & $4(0.6)$ \\
\hline Interferon- $\boldsymbol{\alpha}\left(\mathrm{pg} \cdot \mathrm{mL}^{-1}\right)$ & $0.24(0.24-0.33)$ & $0.56(0.30-1.02)^{\#}$ & $55(7.6)$ \\
\hline Interferon- $\boldsymbol{\beta}\left(\mathrm{pg} \cdot \mathrm{mL}^{-1}\right)$ & $22.7(14.5-34.0)$ & $41.3(22.0-74.1)^{\#}$ & $55(7.6)$ \\
\hline Interferon- $\gamma\left(\mathrm{pg} \cdot \mathrm{mL}^{-1}\right)$ & $2.8(2.8-2.8)$ & $2.8(2.8-4.6)^{\#}$ & $55(7.6)$ \\
\hline Interleukin-1及 $\left(\mathrm{pg} \cdot \mathrm{mL}^{-1}\right)$ & $1.0(1.0-1.0)$ & $1.0(1.0-2.8)^{\#}$ & $55(7.6)$ \\
\hline Interleukin-1 receptor antagonist $\left(\mathrm{pg} \cdot \mathrm{mL}^{-1}\right)$ & $33.0(33.0-551.5)$ & $1280.1(33.0-6244.1)^{\#}$ & $55(7.6)$ \\
\hline Interleukin-2 $\left(\mathrm{pg} \cdot \mathrm{mL}^{-1}\right)$ & $4.4(4.4-4.4)$ & $4.4(4.4-4.4)^{\#}$ & $55(7.6)$ \\
\hline Interleukin-4 (pg $\left.\cdot \mathrm{mL}^{-1}\right)$ & $5.5(5.5-5.5)$ & $5.5(5.5-24.4)^{\#}$ & $55(7.6)$ \\
\hline Interleukin-6 $\left(\mathrm{pg} \cdot \mathrm{mL}^{-1}\right)$ & $40.6(14.6-102.5)$ & $172.0(59.7-748.4)^{\#}$ & $55(7.6)$ \\
\hline Interleukin-8 (pg-mL $\left.{ }^{-1}\right)$ & $3.9(1.9-9.7)$ & $19.8(6.6-46.1)^{\#}$ & $55(7.6)$ \\
\hline Interleukin-10 (pg-mL $\left.{ }^{-1}\right)$ & $0.9(0.7-1.4)$ & $2.2(1.3-4.8)^{\#}$ & $55(7.6)$ \\
\hline Interleukin-12 (pg-mL $\left.{ }^{-1}\right)$ & $1.1(1.1-1.4)$ & $2.2(1.3-3.7)^{\#}$ & $55(7.6)$ \\
\hline Interleukin-13 $\left(\mathrm{pg} \cdot \mathrm{mL}^{-1}\right)$ & $1.3(1.3-1.3)$ & $2.4(1.3-8.8)^{\#}$ & $55(7.6)$ \\
\hline Interleukin-17 (pg-mL $\left.{ }^{-1}\right)$ & $0.57(0.57-0.57)$ & $0.87(0.57-1.86)^{\#}$ & $55(7.6)$ \\
\hline Legionella species & $11(1.9)$ & $3(2.0)$ & $102(14.0)$ \\
\hline Leukocyte count $\left(10^{9}\right.$ cells $\left.\cdot \mathrm{L}^{-1}\right)$ & $11.9(8.7-15.6)$ & $12.2(9.2-15.8)$ & $4(0.6)$ \\
\hline Male $^{\text {I }}$ & $345(60.1)$ & $107(69.9)^{\#}$ & $0(0)$ \\
\hline Monocyte chemoattractant protein $\left(\mathrm{pg} \cdot \mathrm{mL}^{-1}\right)$ & $39.8(25.5-70.1)$ & $66.6(37.2-242.9)^{\#}$ & $55(7.6)$ \\
\hline Neutrophil count $\left(10^{9}\right.$ cells $\left.\cdot \mathrm{L}^{-1}\right)$ & $9.8(6.9-13.2)$ & $10.2(7.4-13.3)$ & $64(9.7)$ \\
\hline Oxygen saturation (\%) & $95(92-96)$ & $94(92-96)$ & $25(3.4)$ \\
\hline Oxygen therapy & $298(51.9)$ & $79(51.6)$ & $6(0.8)$ \\
\hline Pleural effusion" & $65(11.3)$ & $18(11.8)$ & $0(0)$ \\
\hline Pneumonia severity index score & $88(63-111)$ & $98(74-131)^{\#}$ & $0(0)$ \\
\hline Procalcitonin $\left(\mathrm{ng} \cdot \mathrm{mL}^{-1}\right)$ & $0.39(0.16-1.68)$ & $1.14(0.28-10.35)^{\#}$ & $133(18.3)$ \\
\hline Respiratory rate (breaths $\cdot \mathrm{min}^{-1}$ ) & $20(18-24)$ & $20(17-24)$ & $136(18.7)$ \\
\hline Streptococcus pneumoniae & $75(13.1)$ & $31(20.3)^{\#}$ & $104(14.3)$ \\
\hline Tumour necrosis factor- $\alpha\left(\mathrm{pg} \cdot \mathrm{mL}^{-1}\right)$ & $1.8(1.8-1.9)$ & $2.7(1.8-4.0)^{\#}$ & $55(7.6)$ \\
\hline Urea $\left(\mathrm{mmol} \cdot \mathrm{L}^{-1}\right)$ & $6.6(4.8-10.0)$ & $7.9(5.4-13.4)^{\#}$ & $37(5.1)$ \\
\hline
\end{tabular}

Data are shown as median (interquartile range) or $\mathrm{n}(\%)$. \#: statistically significant difference between class 1 and class 2. ": non-class-defining variables (variable not included in latent class analysis). Missing data is $\mathrm{n}(\%)$ for the whole cohort.

Corticosteroids reduced LOS in patients with CAP in the Ovidius trial and in the STEP trial $[16,17]$. An individual patient data meta-analysis enrolling data from six randomized controlled trials comparing corticosteroids with placebo in 1506 patients with CAP, including the Ovidius trial and STEP trial, confirmed that adjunctive treatment with corticosteroids reduced LOS [3]. In this meta-analysis, however, the authors could not identify patient subgroups more likely to benefit from corticosteroids based on PSI score (PSI class 1-3 versus PSI class 4-5), initial C-reactive protein concentration (cut-off $188 \mathrm{mg} \cdot \mathrm{L}^{-1}$ ), initial ICU admission, or systemic inflammatory response syndrome criteria. However, in a clinically heterogeneous condition as CAP, it is unlikely that all patients benefit equally from corticosteroids [9, 14].

In the Ovidius trial, we found that patients assigned to class 2 who were treated with corticosteroids showed a significant reduction in LOS, whereas corticosteroids did not reduce LOS in patients assigned to class 1 . These results suggest that the subgroup of CAP patients with signs of a systemic inflammatory response benefit from corticosteroids and patients with a less pronounced systemic inflammatory response do not. However, these results could not be verified in the STEP cohort, even though PSI score was similar between both cohorts. A possible explanation is that LCAs were performed separately in the Ovidius-TripleP cohort and the STEP cohort and included a different set of class-defining variables for each cohort (figure 1) because available biomarkers differed between both cohorts. Thus, the LCA models 


\begin{tabular}{|c|c|c|c|}
\hline \multicolumn{4}{|l|}{ Ovidius-TripleP cohort } \\
\hline Clinical outcome & Class $1(n=411)$ & Class $2(n=94)$ & $\mathrm{p}$-value \\
\hline Length of stay (days) & $8.0(6.0-12.0)$ & $10.5(6.5-16.0)$ & $<0.01$ \\
\hline ICU admission & $16(3.9 \%)$ & $22(23.4 \%)$ & $<0.01$ \\
\hline In-hospital mortality & $14(3.4 \%)$ & $10(10.6 \%)$ & 0.01 \\
\hline 30-day mortality & $15(3.6 \%)$ & $11(11.7 \%)$ & $<0.01$ \\
\hline Readmission & $29(7.1 \%)$ & $8(8.5 \%)$ & 0.79 \\
\hline \multicolumn{4}{|l|}{ STEP cohort } \\
\hline Clinical outcome & Class $1(n=574)$ & Class $2(n=153)$ & p-value* \\
\hline Length of stay (days) & $7.0(4.0-10.0)$ & $7.0(5.0-12.0)$ & $<0.01$ \\
\hline ICU admission & $28(4.9 \%)$ & $11(7.2 \%)$ & 0.35 \\
\hline In-hospital mortality & $13(2.3 \%)$ & $11(7.2 \%)$ & $<0.01$ \\
\hline 30-day mortality & $18(3.1 \%)$ & $10(6.5 \%)$ & 0.09 \\
\hline Readmission & $30(5.2 \%)$ & $9(5.9 \%)$ & 0.91 \\
\hline
\end{tabular}

were not identical in both cohorts. Furthermore, concentrations of inflammatory biomarkers were higher at baseline in the Ovidius cohort compared to the STEP cohort, indicating a more pronounced inflammatory response in the Ovidius cohort that corticosteroids could inhibit. The reduced three variable model consisting of IL-6, TNF- $\alpha$ and oxygen saturation - showed that the AUC for class assignment was higher in the Ovidius-TripleP cohort as compared to the STEP cohort. This also suggests that the OvidiusTripleP cohort relies more on inflammatory response. Adding to the above, in the STEP cohort, disease severity defined by PSI score was mainly influenced by higher age and more comorbidities, whereas in the Ovidius cohort PSI score was mainly influenced by clinical characteristics and biomarker data indicative of more severe disease. Consequently, clinical variables at baseline did not differ between class 1 and class 2 in the STEP cohort, whereas clinical variables at baseline did differ between classes in the Ovidius cohort.

TABLE 6 Differential response to adjunctive corticosteroid treatment by latent class assignment

Ovidius trial

\begin{tabular}{|c|c|c|c|c|c|}
\hline & \multicolumn{2}{|c|}{ Class $1(n=251)$} & \multicolumn{2}{|c|}{ Class $2(n=52)$} & \multirow[b]{2}{*}{$p$-value* } \\
\hline & Corticosteroid $(n=124)$ & Placebo $(n=128)$ & Corticosteroid $(n=27)$ & Placebo $(n=25)$ & \\
\hline Length of stay (days) & $6.5(5.0-8.5)$ & $7.5(5.5-10.5)$ & $6.5(5.5-10.0)$ & $9.5(5.0-14.5)$ & 0.02 \\
\hline ICU admission & $4(3.2)$ & $4(3.1)$ & $3(11.1)$ & $6(24.0)$ & 0.64 \\
\hline In-hospital mortality & $7(5.6)$ & $3(2.3)$ & $1(3.7)$ & $5(20.0)$ & 0.12 \\
\hline 30-day mortality & $7(5.6)$ & $4(3.1)$ & $2(7.4)$ & $5(20.0)$ & 0.33 \\
\hline Readmission & $6(4.8)$ & $4(3.1)$ & $1(3.7)$ & $3(12.0)$ & 0.56 \\
\hline
\end{tabular}

STEP cohort

\begin{tabular}{|c|c|c|c|c|c|}
\hline & \multicolumn{2}{|c|}{ Class $1(n=574)$} & \multicolumn{2}{|c|}{ Class $2(n=153)$} & \multirow[b]{2}{*}{$p$-value * } \\
\hline & Corticosteroid $(n=285)$ & Placebo $(n=289)$ & Corticosteroid $(n=77)$ & Placebo $(n=76)$ & \\
\hline Length of stay (days) & $6.0(4.0-9.0)$ & $7.0(5.0-10.0)$ & $7.0(4.0-11.0)$ & $8.0(5.0-13.3)$ & 0.46 \\
\hline ICU admission & $11(3.9)$ & $17(5.9)$ & $6(7.8)$ & $5(6.6)$ & 0.61 \\
\hline In-hospital mortality & $8(2.8)$ & $5(1.7)$ & $5(6.5)$ & $6(7.9)$ & 0.71 \\
\hline 30-day mortality & $11(3.9)$ & $7(2.4)$ & $4(5.2)$ & $6(7.9)$ & 0.50 \\
\hline Readmission & $21(7.4)$ & $9(3.1)$ & $5(6.5)$ & $4(5.3)$ & 0.69 \\
\hline
\end{tabular}

Data are $\mathrm{n}(\%)$ or median (interquartile range). * $\mathrm{p}$-value: for interaction between class assignment and corticosteroid treatment. ICU: intensive care unit; STEP: Steroids in Pneumonia. 
Other explanations might be the difference in corticosteroid therapy (dexamethasone versus prednisolone) or the shorter LOS in the STEP cohort (median 8.5; 6.0-13.0 days in Ovidius cohort versus 7.0; 4.0 10.0 days in STEP cohort) making potential differences between classes in the STEP cohort more difficult to detect.

Inflammatory biomarkers contributed more to the determination of classes than clinical data, including C-reactive protein, procalcitonin or leukocyte count. These results suggest that the inflammatory biomarkers were able to identify aspects of CAP pathophysiology that otherwise remained hidden in routinely collected clinical data.

This study has several limitations. First, LCA model selection and interpretation often involves a level of subjectivity [19]. We decided to select a two-class model instead of more classes based on clinical interpretability and the number of patients assigned to the smallest class. Hypothetically, a third class or even a fourth class could have been forced in by generating a smaller cluster of patients with a more extreme set of variables. However, a three-or-more-class model did not result in additional groups with more extreme variables, but in mixed classes without a coherent clinical pattern. Second, we assumed patients in class 2 to have a systemic inflammatory response and patients in class 1 to have a more controlled inflammatory response based on distribution of inflammatory biomarkers in plasma. We did not measure the pulmonary response and therefore do not know whether inflammation is indeed contained locally in patients assigned to class 1 . We refrained from using terms as hyperinflammatory or hypoinflammatory, previously proposed in subgroups of patients with acute respiratory distress syndrome, as all patients are admitted because of CAP, which can hardly be considered a hypoinflammatory condition [20,21]. Third, this is a secondary analysis which requires prospective validation before definitive conclusions regarding patient subgroup identification and adjunctive corticosteroid treatment can be drawn. Fourth, LOS was calculated from day of hospital admission to day of discharge or day of in-hospital death. Thus, LOS was underestimated in patients that died during hospital admission. However, in both cohorts, in-hospital mortality rate was higher in class 2 as compared to class 1 . If reported LOS were an underestimation, this would mainly be the case in class 2 and the difference in LOS between classes would be even larger than reported. Fifth, the clinical and biomarker data used in this analysis was limited to the data available for both cohorts and to data obtained at time of hospital admission. As the aim of data collection for the original studies was to calculate the PSI score, clinical data used in the LCA resembled the PSI score to some extent and PSI score differed significantly between class 1 and class 2 in both cohorts. However, the classes identified by LCA were largely based on biomarker data and thus captured different subgroups of patients than classes based on PSI score only. Lastly, because data was obtained at time of hospital admission, it is unknown whether identified classes remained stable later during the course of CAP.

To our knowledge, this is the first study that identified CAP subgroups through LCA. Because the present study is a proof-of-concept study, our results are not directly applicable for daily clinical practice. Future studies should include validation of our findings in a third independent cohort, after which a clinically useful model with a limited number of variables should be developed to ensure applicability. Lastly, validation of these clinical models in predicting response to treatment should be assessed in prospective studies.

In conclusion, we identified two classes of CAP patients with different clinical characteristics, inflammatory profiles and clinical outcomes in two independent cohorts. Furthermore, in the Ovidius trial, adjunctive treatment with corticosteroids reduced LOS only in the patients assigned to class 2 and not in the patients assigned to class 1 . Given the different response to adjunctive treatment in subgroups in the Ovidius cohort, identification of subgroups might provide a useful basis for improved patient selection in future clinical trials.

Author contributions: E. Wittermans and P.A. van der Zee participated in data cleaning and analysis, interpreted the data, and drafted and revised the manuscript. H. Qi participated in data cleaning and analysis, interpreted the data, and revised the manuscript. E.M.W. van de Garde, C.A. Blum, M. Christ-Crain, D. Gommers, J.C. Grutters, G.P. Voorn, W.J.W. Bos and H. Enderman interpreted the data and revised the manuscript. All authors had access to the raw data. All authors provided final approval of the version to be published.

Provenance: Submitted article, peer reviewed.

Data sharing: Individual participant data that underlie the results reported in this article, after de-identification, and a dictionary defining each field in the dataset will be made available after approval of the proposal by the study group. Requests should be directed tow.bos@antoniusziekenhuis.nl (on behalf of the study group) along with an analysis proposal; data requestors will need to sign a data access agreement. 
Conflict of interest: E. Wittermans has nothing to disclose. P.A. van der Zee has nothing to disclose. H. Qi has nothing to disclose. E.M.W. van de Garde has nothing to disclose. C.A. Blum reports grants from the Helmut Horten Foundation, Switzerland, and Novo Nordisk, outside the submitted work. M. Christ-Crain has nothing to disclose. D. Gommers reports speaker fees and travel expenses from Drager and Maquet, personal fees and other support for a medical advisory board from 2009 to 2012 from GE Healthcare, and personal fees and other support for a medical advisory board from 2015 to 2018 from Novalung, outside the submitted work. J.C. Grutters has nothing to disclose. G.P. Voorn has nothing to disclose. W.J.W. Bos has nothing to disclose. H. Endeman has nothing to disclose.

Support statement: This study was supported by the St Antonius Research Fund. Funding information for this article has been deposited with the Crossref Funder Registry.

\section{References}

1 Metlay JP, Waterer GW, Long AC, et al. Diagnosis and treatment of adults with community-acquired pneumonia. an official clinical practice guideline of the American Thoracic Society and Infectious Diseases Society of America. Am J Respir Crit Care Med 2019; 200: e45-e67.

2 Troeger C, Blacker BF, Khalil IA, et al. Estimates of the global, regional, and national morbidity, mortality, and aetiologies of lower respiratory infections in 195 countries, 1990-2016: a systematic analysis for the Global Burden of Disease Study 2016. Lancet Infect Dis 2018; 18: 1191-1210.

3 Briel M, Spoorenberg SMC, Snijders D, et al. Corticosteroids in patients hospitalized with community-acquired pneumonia: systematic review and individual patient data metaanalysis. Clin Infect Dis 2018; 66: 346-354.

4 Bermejo-Martin JF, Almansa R, Martin-Fernandez M, et al. Immunological profiling to assess disease severity and prognosis in community-acquired pneumonia. Lancet Resp Med 2017; 5: E35-E36.

5 Rhen T, Cidlowski JA. Antiinflammatory action of glucocorticoids new-mechanisms for old drugs. $N$ Engl J Med 2005; 353: 1711-1723.

6 Chalmers JD. Corticosteroids for community-acquired pneumonia: a critical view of the evidence. Eur Respir J 2016; 48: 984-986.

7 Meijvis SCA, van de Garde EMW, Rijkers GT, et al. Treatment with anti-inflammatory drugs in community-acquired pneumonia. J Intern Med 2012; 272: 25-35.

8 Torres A, Sibila O, Ferrer M, et al. Effect of corticosteroids on treatment failure among hospitalized patients with severe community-acquired pneumonia and high inflammatory response: a randomized clinical trial. JAMA 2015; 313: 677-686.

9 Remmelts $\mathrm{HH}$, Meijvis SC, Heijligenberg R, et al. Biomarkers define the clinical response to dexamethasone in community-acquired pneumonia. J Infect 2012; 65: 25-31.

10 Urwyler SA, Blum CA, Coslovsky M, et al. Cytokines and Cortisol - predictors of treatment response to corticosteroids in community-acquired pneumonia? J Intern Med 2019; 286: 75-87.

11 Scicluna BP, van Vught LA, Zwinderman AH, et al. Classification of patients with sepsis according to blood genomic endotype: a prospective cohort study. Lancet Resp Med 2017; 5: 816-826.

12 Calfee CS, Delucchi K, Parsons PE, et al. Subphenotypes in acute respiratory distress syndrome: latent class analysis of data from two randomised controlled trials. Lancet Respir Med 2014; 2: 611-620.

13 Famous KR, Delucchi K, Ware LB, et al. Acute respiratory distress syndrome subphenotypes respond differently to randomized fluid management strategy. Am J Respir Crit Care Med 2017; 195: 331-338.

14 Prescott HC, Calfee CS, Thompson BT, et al. Toward smarter lumping and smarter splitting: rethinking strategies for sepsis and acute respiratory distress syndrome clinical trial design. Am J Respir Crit Care Med 2016; 194: 147-155.

15 Endeman H, Meijvis SC, Rijkers GT, et al. Systemic cytokine response in patients with community-acquired pneumonia. Eur Respir J 2011; 37: 1431-1438.

16 Meijvis SC, Hardeman H, Remmelts $\mathrm{HH}$, et al. Dexamethasone and length of hospital stay in patients with community-acquired pneumonia: a randomised, double-blind, placebo-controlled trial. Lancet 2011; 377: 2023-2030.

17 Blum CA, Nigro N, Briel M, et al. Adjunct prednisone therapy for patients with community-acquired pneumonia: a multicentre, double-blind, randomised, placebo-controlled trial. Lancet 2015; 385: 1511-1518.

18 Cham H, Reshetnyak E, Rosenfeld B, et al. Full information maximum likelihood estimation for latent variable interactions with incomplete indicators. Multivariate Behav Res 2017; 52: 12-30.

19 Al Sallakh MA, Rodgers SE, Lyons RA, et al. Identifying patients with asthma-chronic obstructive pulmonary disease overlap syndrome using latent class analysis of electronic health record data: a study protocol. NPJ Prim Care Respir Med 2018; 28: 22.

20 Sinha P, Calfee CS. Phenotypes in acute respiratory distress syndrome: moving towards precision medicine. Curr Opin Crit Care 2019; 25: 12-20.

21 Calfee CS, Delucchi KL, Sinha P, et al. Acute respiratory distress syndrome subphenotypes and differential response to simvastatin: secondary analysis of a randomised controlled trial. Lancet Respir Med 2018; 6: 691-698. 